The Astrophysical Journal, 281 : 1-8, 1984 June 1

(C) 1984. The American Astronomical Society. All rights reserved. Printed in U.S.A.

\title{
SELF-SIMILAR GRAVITATIONAL COLLAPSE IN AN EXPANDING UNIVERSE ${ }^{1}$
}

\author{
James A. Fillmore and Peter Goldreich \\ California Institute of Technology \\ Received 1983 October 10; accepted 1983 December 5
}

\begin{abstract}
We derive similarity solutions which describe the collapse of cold, collisionless matter in a perturbed Einstein-de Sitter universe. We obtain three classes of solutions, one each with planar, cylindrical, and spherical symmetry. Our solutions can be computed to arbitrary accuracy, and they follow the development of structure in both the linear and nonlinear regimes.
\end{abstract}

Subject headings: cosmology - relativity

\section{INTRODUCTION}

Understanding the formation of structure in an expanding universe is one of the outstanding problems of modern cosmology. Zel'dovich (1970) was the first to emphasize that anisotropic collapse characterizes the evolution of structure in a universe filled with pressureless matter. High-density regions exhibiting approximate planar, cylindrical, and spherical symmetry commonly form in three-dimensional $N$-body simulations (Klypin and Shandarin 1983; Frenk, White, and Davis 1983; Centrella and Melott 1983).

We investigate self-similar collapse solutions with planar, cylindrical, and spherical symmetry. Study of these solutions provides considerable insight into the more complicated results obtained from numerical simulations. Of course, the restriction to specialized initial conditions is the price we must pay to obtain similarity solutions. It is convenient to treat the three symmetries in parallel. We use the parameter $n$ to distinguish among them; $n$ equals 1,2 , and 3 for planar, cylindrical, and spherical symmetry, respectively.

The plan of the paper is as follows. In $\S$ II we derive the equations which govern the evolution of the similarity solutions. In § III we obtain analytic expressions for the asymptotic properties of the solutions. The results of numerical integrations of the similarity equations are presented in $\S \mathrm{IV}$. The relation of our solutions to previous work is discussed in $\S \mathrm{V}$. The scale factors used for the three different symmetries are calculated in the Appendix.

\section{DERIVATION OF SIMILARITY EQUATIONS}

As the scale of the perturbations which we are investigating is always small compared with that of the horizon, Newtonian cosmology is an adequate approximation (Peebles 1980). The equation of motion of a test particle reads

$$
\frac{d^{2} \boldsymbol{r}(t)}{d t^{2}}=-\frac{4 \pi}{3} G \rho_{b}(t) \boldsymbol{r}+\delta \boldsymbol{g}(\boldsymbol{r}, t) .
$$

The first term on the right-hand side of the equation is the deceleration due to the unperturbed Einstein-de Sitter background density

$$
\rho_{b}(t)=\frac{1}{6 \pi G t^{2}}
$$

\footnotetext{
${ }^{1}$ Contribution No. 3992 of the Division of Geological and Planetary Sciences, California Institute of Technology.
}

The second term is the peculiar acceleration caused by the perturbation density

$$
\delta \rho(\boldsymbol{r}, t)=\rho(\boldsymbol{r}, t)-\rho_{b}(t) .
$$

The position of a particle is denoted by its distance from the center of symmetry $x$. For planar, cylindrical, and spherical symmetry, $x$ denotes the distance from the symmetry plane $z$, the distance from the symmetry axis $\varpi$, the distance from the symmetry point $r$.

We define the mass $M(x, t)$ and excess mass $\delta M(x, t)$ within $x$ by

$$
M(x, t)=\int_{0}^{x} d x^{\prime} x^{\prime n-1} \rho\left(x^{\prime}, t\right) .
$$

For planar symmetry,

$$
M(z, t)=\int_{0}^{z} d z^{\prime} \rho\left(z^{\prime}, t\right),
$$

the mass per unit area. For cylindrical symmetry,

$$
M(\varpi, t)=\int_{0}^{\varpi} d \varpi^{\prime} \varpi^{\prime} \rho\left(\varpi^{\prime}, t\right),
$$

the mass per unit length per unit angle. For spherical symmetry,

$$
M(r, t)=\int_{0}^{r} d r^{\prime} r^{\prime 2} \rho\left(r^{\prime}, t\right),
$$

the mass per unit solid angle.

The peculiar acceleration is related to $\delta M$ by

$$
\delta g(x, t)=-\frac{4 \pi G \delta M}{x^{n-1}}
$$

Inserting this expression into the equation of motion yields

$$
\frac{d^{2} x}{d t^{2}}=-\frac{2}{9} \frac{x}{t^{2}}-\frac{4 \pi G \delta M}{x^{n-1}}=\frac{2}{9} \frac{(3-n)}{n} \frac{x}{t^{2}}-\frac{4 \pi G M}{x^{n-1}} .
$$

We choose initial conditions such that at time $t_{i}$ the unperturbed Hubble law

$$
\frac{d \boldsymbol{r}}{d t}=\frac{2}{3} \frac{\boldsymbol{r}}{t}
$$

is exactly satisfied. We can imagine that $t_{i}$ corresponds to the time of decoupling of radiation and matter. The initial position 
of a test particle is denoted either by its initial distance from the center of symmetry, $x_{i}$, or by the initial mass, $M_{i}$, between it and the center of symmetry; $M_{i} \equiv M\left(x_{i}, t_{i}\right)$.

The initial perturbation is characterized by the profile of $\delta M_{i}$. We express $\delta M_{i}$ as a function of $M_{i}$. The search for similarity solutions dictates that the initial perturbation be scale free; thus $\delta M_{i}\left(M_{i}\right)$ must be a power law. We write

$$
\delta \equiv \frac{\delta M_{i}}{M_{i}}=\left(\frac{M_{i}}{M_{0}}\right)^{-\epsilon},
$$

where $M_{0}$ is a reference mass; the corresponding reference position is $x_{0}$. The parameter $\epsilon$ is restricted to values between zero and unity by the requirements that the initial mass perturbation increase and the initial density perturbation decrease away from the center of symmetry.

Our choice of initial conditions is clearly incompatible with exact self-similarity; at $t_{i}$ there is no collapsed material although the overdensity in the inner regions diverges. It is a plausible assumption that the trajectories of the material with small $\delta$ will approach a self-similar solution. This would be satisfactory since for $t \gg t_{i}$ almost all of the collapsed material has small $\delta$.

Because the initial density exceeds the critical value $\rho_{b}(t)$, the trajectory of each particle will ultimately oscillate through the center of symmetry. We refer to the events corresponding to the local maxima of $x\left(t, M_{i}\right)$ as apapsis passages. The first apapsis passage is of special significance, and we reserve the name of turnaround for it. The turnaround distance and time are denoted by $x_{*}\left(M_{i}\right)$ and $t_{*}\left(M_{i}\right)$. Prior to reaching turnaround, a particle does not cross the orbits of other particles. Thus, for $t<t_{*}$,

$$
\frac{M(x, t)}{M_{i}}=\left(\frac{t_{i}}{t}\right)^{2(3-n) / 3},
$$

where the $\left(t_{i} / t\right)^{2(3-n) / 3}$ factor is a consequence of the unperturbed Hubble expansion along $3-n$ of the coordinate axes. Using this simple expression for $M(x, t)$, we solve the equation of motion in the interval $t_{i}<t \leq t_{*}$ (cf. Appendix), to obtain

$$
\begin{aligned}
\frac{x_{*}}{x_{i}} & =\frac{C_{x}}{\delta}, \\
\frac{t_{*}}{t_{i}} & =\left(\frac{C_{t}}{\delta}\right)^{3 / 2} .
\end{aligned}
$$

Equations (13) and (14) are valid to lowest order in $\delta \ll 1$. The appropriate values of $C_{x}$ and $C_{t}$ for the three symmetries are

$$
\begin{aligned}
& C_{x}=\frac{5}{12}, 0.74, \quad 1 ; \\
& C_{t}=\frac{5}{6}, \quad 1.39, \quad\left(\frac{3 \pi}{4}\right)^{2 / 3} .
\end{aligned}
$$

Inserting equation (11) into equations (13) and (14) yields

$$
\begin{aligned}
\frac{x_{*}}{x_{i}} & =C_{x}\left(\frac{M_{i}}{M_{0}}\right)^{\epsilon}, \\
\frac{t_{*}}{t_{i}} & =C_{t}^{3 / 2}\left(\frac{M_{i}}{M_{0}}\right)^{3 \epsilon / 2} .
\end{aligned}
$$

With the aid of equation (4), we find

$$
x_{*}=C_{x}\left[\frac{n M_{0}}{\rho_{b}\left(t_{i}\right)}\right]^{1 / n}\left(\frac{M_{i}}{M_{0}}\right)^{\epsilon+1 / n} .
$$

We denote the current turnaround distance by $X(t)$ and the corresponding initial mass by $M_{t}$. From equations (16) and (17) it follows that

$$
\begin{gathered}
X(t)=\frac{C_{x}}{C_{t}^{1+1 / n \epsilon}}\left[\frac{n M_{0}}{\rho_{b}\left(t_{i}\right)}\right]^{1 / n}\left(\frac{t}{t_{i}}\right)^{2 / 3+2 /(3 n \epsilon)}, \\
\frac{M(X, t)}{M_{0}}=\frac{1}{C_{t}^{1 / \epsilon}}\left(\frac{t}{t_{i}}\right)^{2 /(3 \epsilon)-2(3-n) / 3} .
\end{gathered}
$$

We define dimensionless coordinate and time variables

$$
\lambda \equiv \frac{x}{x_{*}}, \quad \tau \equiv \frac{t}{t_{*}} .
$$

We now prove that, expressed in terms of these scaled variables, the equation of motion is independent of $M_{i}$.

Sometime after turnaround a particle crosses the trajectory of other particles, so the simple relation (eq. [12]) for $M(x, t)$ is no longer valid. The major step in the derivation of the similarity equation is to express $M(x, t)$ as a functional of $\lambda(\tau)$. We appeal to self-similarity and write

$$
M(x, t) \equiv M(X, t) \mathscr{M}(x / X),
$$

where $\mathscr{M}$ is the dimensionless mass profile function given by

$$
\mathscr{M}\left(\frac{x}{X}\right) \equiv \int_{0}^{M_{t}} \frac{d M_{i}^{\prime}}{M_{t}} H\left[x\left(t ; M_{i}\right)-x\left(t ; M_{i}^{\prime}\right)\right],
$$

for $x / X \leq 1$. Here $H[u]$ is the Heaviside function; $H[u]=0$ for $u<0$, and $H[u]=1$ for $u \geq 0$. Changing the variable of integration from $M_{i}$ to $\tau$ with the help of equation (16) yields

$$
\mathscr{M}\left(\frac{\lambda}{\Lambda}\right)=\frac{2}{3 \epsilon} \int_{1}^{\infty} \frac{d \xi}{\xi^{1+2 /(3 \epsilon)}} H\left[\frac{\lambda}{\Lambda}-\frac{\lambda(\xi)}{\Lambda(\xi)}\right],
$$

where $\Lambda$ is defined by

$$
\Lambda(\tau) \equiv \tau^{2 / 3+2 /(3 n \epsilon)} .
$$

Finally, we use equations (17), (18), and (20) to rewrite the equation of motion (9) in terms of the scaled voriables:

$$
\frac{d^{2} \lambda}{d \tau^{2}}=\frac{2(3-n)}{9 n} \frac{\lambda}{\tau^{2}}-\frac{2}{3 n}\left(\frac{C_{t}}{C_{x}}\right)^{n} \frac{\tau^{2 /(3 \epsilon)-2(3-n) / 3}}{\lambda^{n-1}} \mathscr{M}\left(\frac{\lambda}{\Lambda}\right) .
$$

Equation (25) governs the evolution of the similarity solutions for all values of $\tau$. It requires the boundary conditions

$$
\lambda(1)=1, \quad \frac{d \lambda(1)}{d \tau}=0
$$

which reflect that turnaround occurs at $\tau=1, \lambda=1$.

Proper application of Newtonian cosmology requires that the linear dimensions of the structures be small compared with the distance to the horizon. This restricts the interval of time over which the similarity solutions are valid in two respects. First, it implies that the planar and cylindrical solutions must be thought of as possessing edges along $3-n$ symmetry directions. Our analysis neglects edge effects, but they must become important when the turnaround distance approaches the system's dimensions along the symmetry directions. This is inevitable since, for all cases, the turnaround distance grows more rapidly than $t^{2 / 3}$, the rate of expansion along the symmetry directions. Second, for spherical solutions with $\epsilon<\frac{2}{3}$, the turnaround distance grows faster than $t$ and must eventually approach the horizon scale. 
III. ASYMPTOTIC BEHAVIOR OF SIMILARITY SOLUTIONS

The similarity solutions describe a basic type of motion. There is an initial period of expansion which ends at turnaround. After turnaround, the trajectory oscillates through the center of symmetry. With the passage of time, the ratio of the apapsis distance to the current turnaround distance decreases as does the ratio of the oscillation period to the time scale for halo evolution. Thus the trajectory is asymptotically buried ever more deeply in the halo of collapsed material.

The above picture implies that there is an adiabatic invariant associated with buried trajectories. Furthermore, the scalefree nature of the similarity solutions suggests that the mass profile in the halo might be approximated by a power law in the distance from the center of symmetry. These features enable us to deduce the asymptotic properties of the similarity solutions.

We parameterize the halo mass profile by

$$
M(x, t)=\kappa(t) x^{\gamma},
$$

and the variation of the apapsis distance $x_{a}$ by

$$
\frac{x_{a}}{x_{*}}=\left(\frac{t}{t_{*}}\right)^{q} \text {. }
$$

Our goal is to relate $\gamma$ and $q$ to $\epsilon$

The equation of motion of a halo particle may be written as

$$
\frac{d^{2} x}{d t^{2}}=-4 \pi G \kappa(t) x^{\gamma-n+1}
$$

Orbits for which $x_{a} \ll X$ have periods which are much shorter than $t$. Thus it is a good approximation to treat $\kappa$ as constant over one orbit period. For constant $\kappa$, equation (29) has the energy integral

$$
\left(\frac{d x}{d t}\right)^{2}=\frac{8 \pi G \kappa(t)}{(\gamma-n+2)}\left(x_{a}^{\gamma-n+2}-x^{\gamma-n+2}\right) .
$$

The gradual variation of the apapsis distance due to the time dependence of $\kappa$ is obtained from the action

$$
\begin{aligned}
J & =4 \int_{0}^{x_{a}} d x\left(\frac{d x}{d t}\right) \\
& =8\left[\frac{2 \pi G \kappa(t)}{\gamma-n+2}\right]^{1 / 2} x_{a}^{(\gamma-n+4) / 2} \int_{0}^{1} d u\left(1-u^{\gamma-n+2}\right)^{1 / 2},
\end{aligned}
$$

which is an adiabatic invariant. We define the auxiliary parameter $s$ by $\kappa(t)=c t^{-s}$. Thus $q=s /(\gamma-n+4)$. We use equations (18), (19), and (27) to express $s$ in terms of $\gamma$ and $\epsilon$ as

$$
s=\frac{2}{3 \epsilon}\left[\frac{(\gamma-n)}{n}+(\gamma-n+3) \epsilon\right] .
$$

Hence

$$
q=\frac{2}{3 \epsilon(\gamma-n+4)}\left[\frac{(\gamma-n)}{n}+(\gamma-n+3) \epsilon\right] .
$$

We define $P\left(x / x_{a}\right)$ to be the fraction of time a particle with apapsis $x_{a}$ spends inside $x$ :

$$
\begin{aligned}
& P(v)=\frac{I(v)}{I(1)} \quad(v \leq 1), \\
& P(v)=1 \quad(v>1),
\end{aligned}
$$

where

$$
I(v) \equiv \int_{0}^{v} \frac{d u}{\left(1-u^{\gamma-n+2}\right)^{1 / 2}} .
$$

Self-consistency demands that

$$
\left(\frac{x}{X}\right)^{\gamma}=\frac{M(x, t)}{M(X, t)}=\int_{0}^{M_{t}} \frac{d M_{i}}{M_{t}} P\left[\frac{x}{x_{a}\left(t ; M_{i}\right)}\right] .
$$

Transforming the integration variable from $M_{i}$ to $u \equiv x / x_{a}$ using equations (17), (18), and (28), we obtain

$$
\left(\frac{x}{X}\right)^{\gamma-p}=\frac{1}{p} \int_{x / X}^{\infty} \frac{d u}{u^{1+p}} P(u)
$$

where

$$
p=\frac{2 n}{2+n(2-3 q) \epsilon} .
$$

Now we have arrived at the crucial point. For $u \ll 1$, $P(u) \approx u / I(1)$. Thus the integral in equation (37) converges or diverges as $x / X$ approaches 0 according to whether $p$ is less than or greater than 1 . In the former case,

$$
p \leq 1, \quad \gamma=p
$$

whereas, in the latter,

$$
p \geq 1, \quad \gamma=1 \text {. }
$$

The physical distinction between the two cases is clear. For $p<1$, the mass in the inner halo is dominated by particles with small ' apapsides, $x_{a} / X \ll 1$, which passed their turnaround events a long time ago. On the other hand, for $p>1$, the mass in the inner halo comes from particles whose apapsides are spread throughout the halo.

Completing the allowable solutions with the aid of equations (33) and (38), we find

$n=1$ :

$$
\gamma=p=\frac{3}{3+\epsilon} \leq 1, \quad q=\frac{4}{9}
$$

$n=2$ :

$$
\gamma=1, \quad p=\frac{3}{2+\epsilon} \geq 1, \quad q=\frac{4 \epsilon-1}{9 \epsilon}
$$

$n=3$ :

$$
\begin{array}{ll}
\gamma=1, \quad p=\frac{6}{4+3 \epsilon} \geq 1, \quad q=\frac{3 \epsilon-2}{9 \epsilon}, & \text { for } \epsilon \leq \frac{2}{3} \\
\gamma=p=\frac{3}{1+3 \epsilon} \leq 1, \quad q=0, & \text { for } \epsilon \geq \frac{2}{3} .
\end{array}
$$

\section{NUMERICAL INTEGRATION OF SIMILARITY EQUATIONS}

The similarity solutions are determined by numerical integration of the second-order differential equation (25) subject to the boundary conditions given by equations (26). Backward integration is straightforward because $\mathscr{M}=\tau^{-2 /(3 \epsilon)}$ for $\tau \leq 1$. For $\tau \geq 1, \mathscr{M}$ is a functional of $\lambda$, so an iterative method must be employed. Following an initial guess for $\mathscr{M}$, we alternately integrate equation (25) over the selected domain of $\tau \geq 1$ and then compute $\mathscr{M}$ over the same domain of $\tau$ from equation (23). This procedure is continued until the desired level of convergence is achieved. The solutions are quite robust, and 


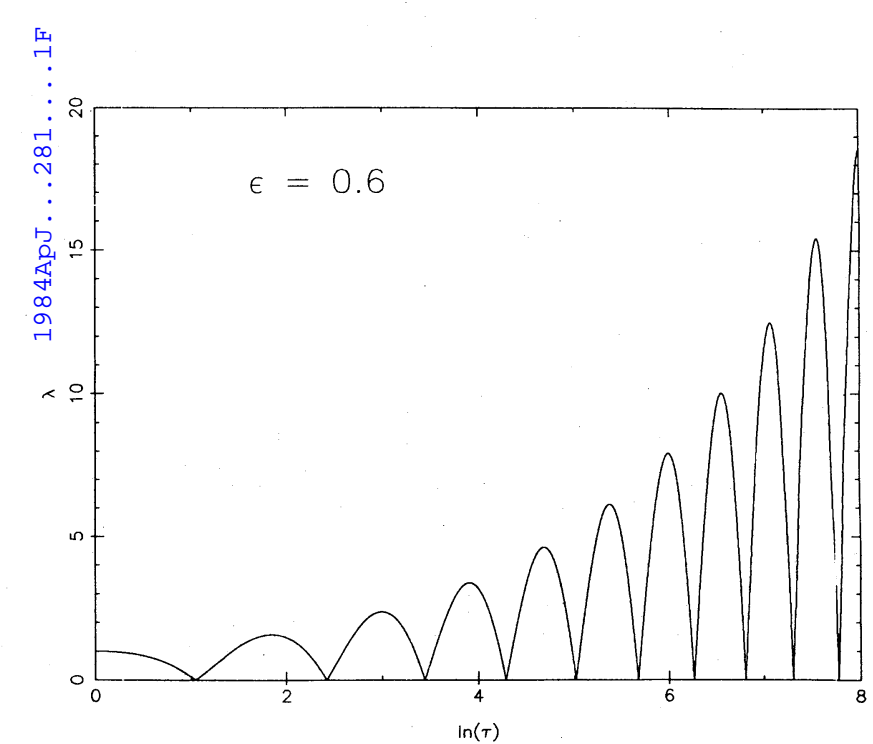

FIG. 1

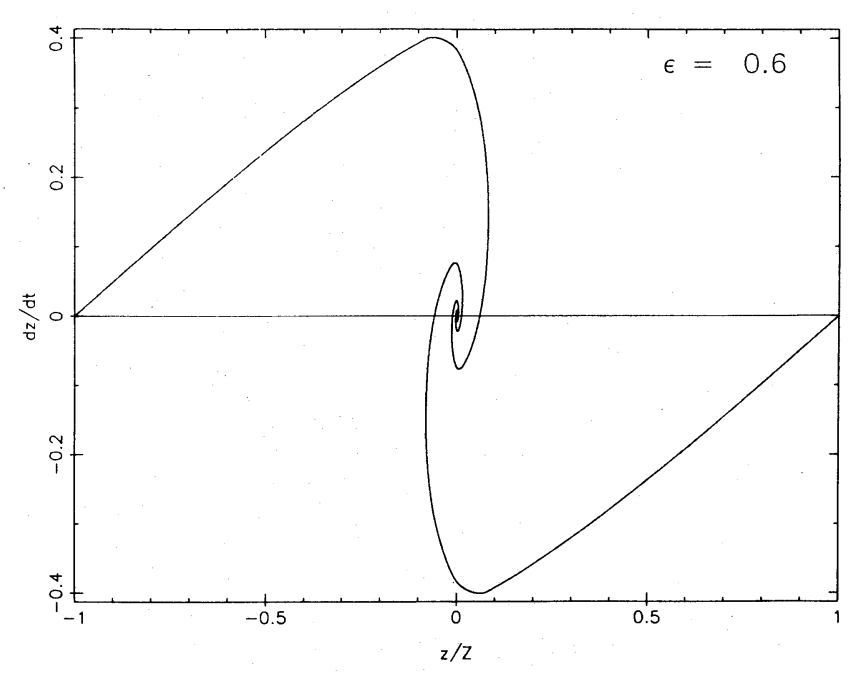

FIG. 2

FIG. 1.-Planar symmetry: particle trajectory for $\epsilon=0.6$

FIG. 2.-Planar symmetry: instantaneous location of all particles in phase space for $\epsilon=0.6$.

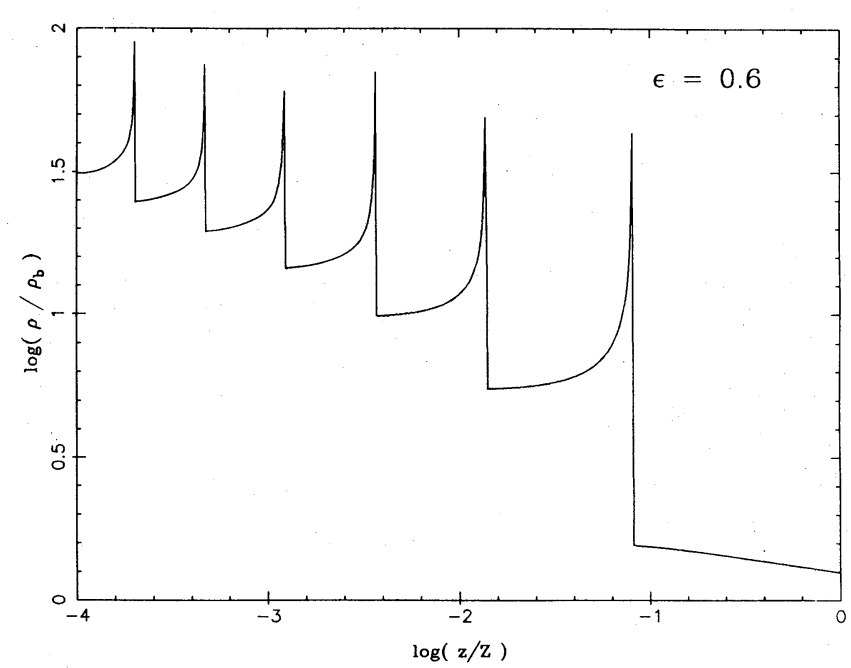

FIG. 3

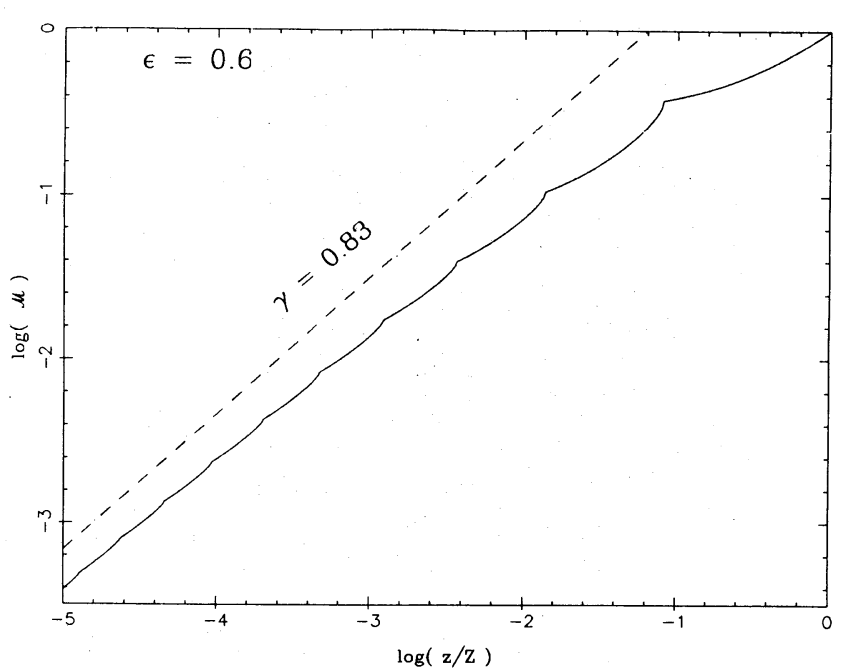

FIG. 4

FIG. 3.-Planar symmetry: ratio of actual to background density for $\epsilon=0.6$.

FIG. 4.-Planar symmetry: profile of mass per unit area for $\epsilon=0.6$. Dashed line shows predicted power-law slope.

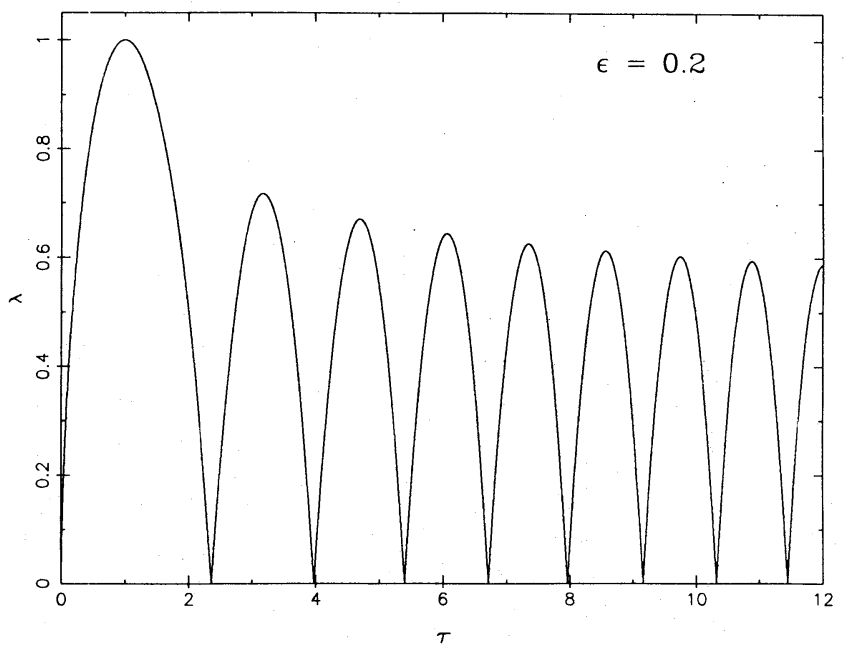

FIG. $5 a$

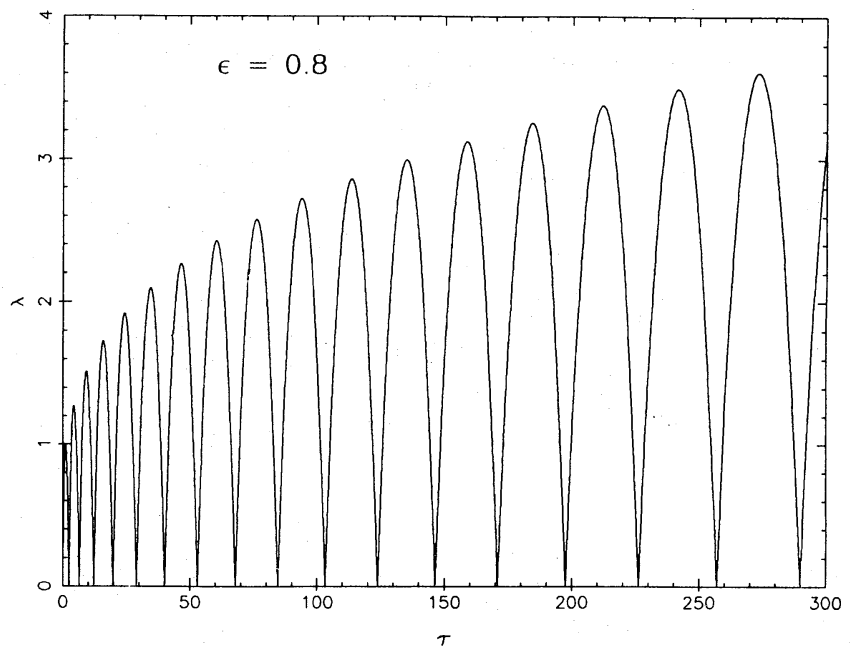

FIG. $5 b$

FIG. 5.-Cylindrical symmetry: particle trajectory for $(a) \epsilon=0.2,(b) \epsilon=0.8$ 


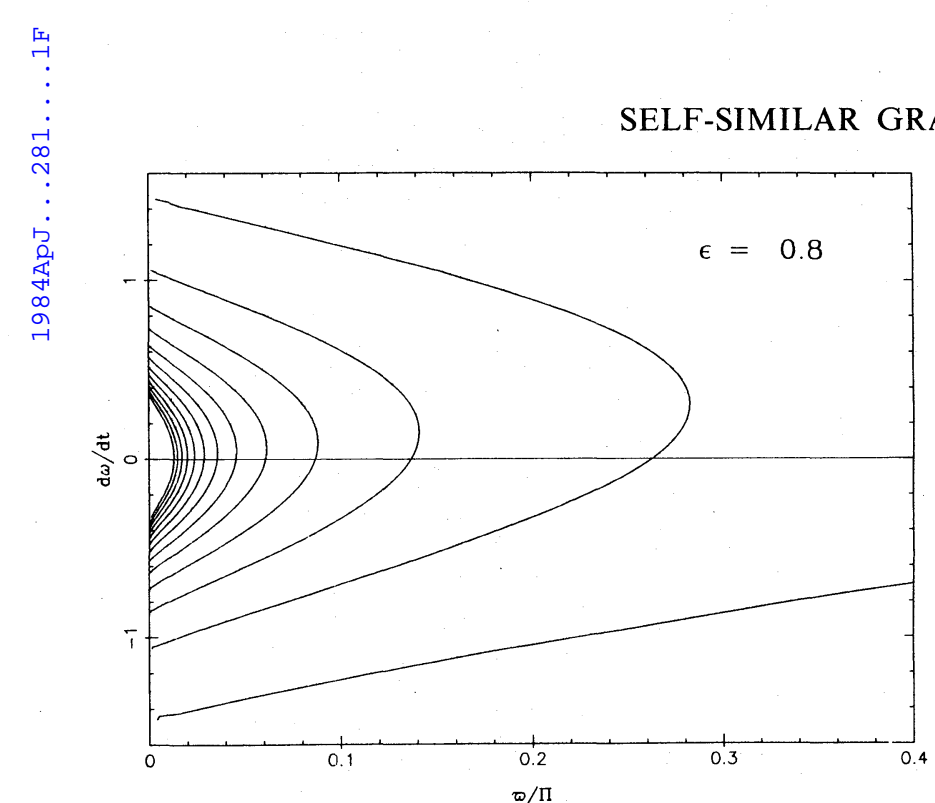

FIG. 6-Cylindrical symmetry: instantaneous location of all particles in phase space for $\epsilon=0.8$.

almost any monotonic function of $\lambda / \Lambda$ which satisfies $\mathscr{M}(0)=0$ and $\mathscr{M}(1)=1$ provides an adequate input guess.

The integral giving $\mathscr{M}$ is calculated at a discrete grid of points; typically 500 are used. It is evaluated between these points by linear interpolation. A higher order spline interpolation is unsuitable because $\mathscr{M}$ is not a smooth function. The ratio of the particle's apapsis distance to the current turnaround distance, $\lambda_{a} / \Lambda$, decreases with increasing $\tau$. The similarity solution is generally determined out to the value of $\tau$ at which $\lambda_{a} / \Lambda$ falls below the lowest grid point.

At small values of $\lambda$ the acceleration is proportional to $\lambda^{(\gamma-n+1)}$. Thus, near the origin it vanishes in the planar case, equals unity in the cylindrical case, and diverges in the spherical case. In the last two cases the trajectory is integrated analytically through the origin.

The principal features of each solution are illustrated in a sequence of four figures. There are three such sequences (Figs. 1-12), one for each symmetry. The first figure of each sequence depicts the oscillatory behavior of $\lambda(\tau)$. The second figure of

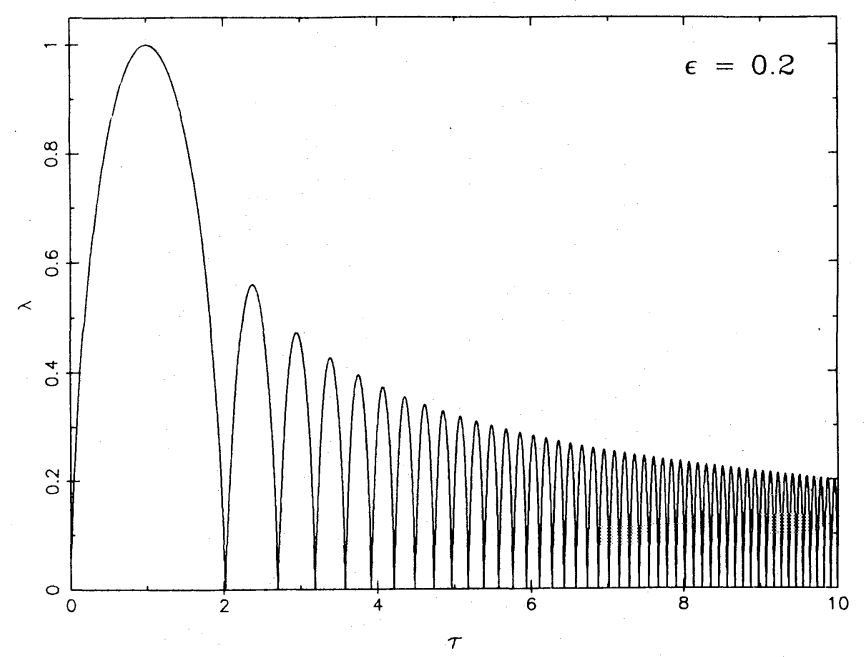

FIG. $9 a$

FIG. 9.-Spherical symmetry: particle trajectory for $(a) \epsilon=0.2$, and $(b) \epsilon=1.0$

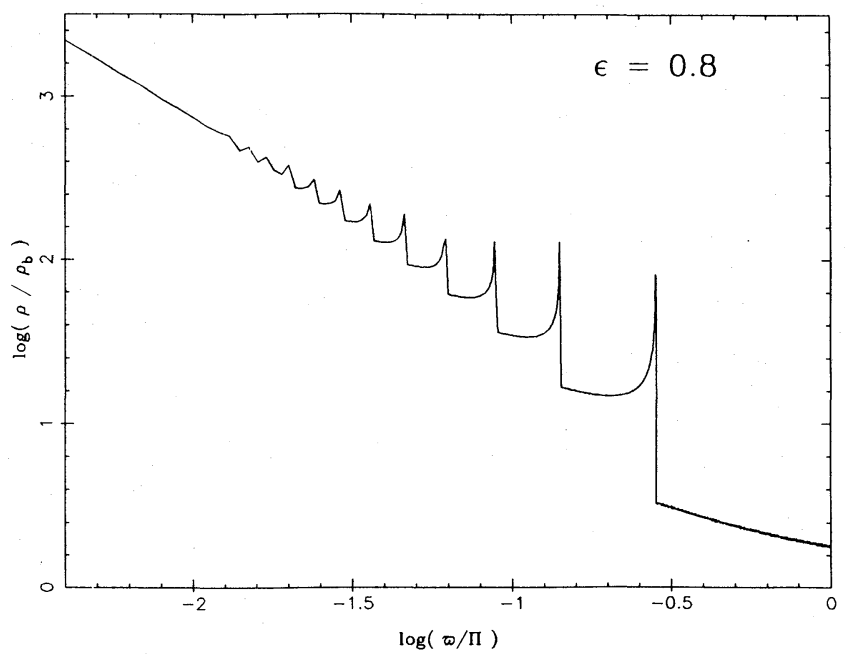

FIG. 7.-Cylindrical symmetry: ratio of actual to background density for $\epsilon=0.8$.

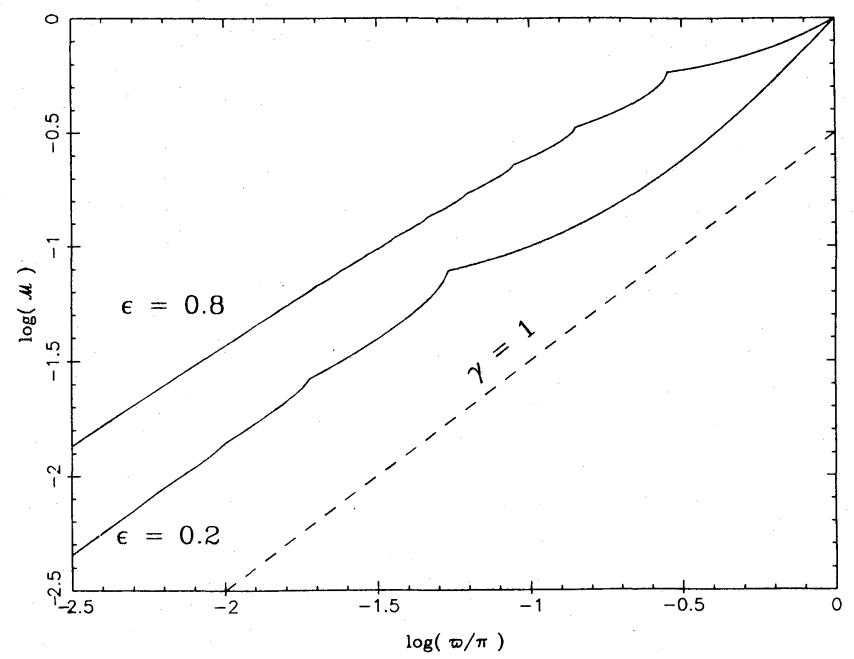

FIG. 8.-Cylindrical symmetry: profiles of mass per unit length per unit angle for $\epsilon=0.2$ and 0.8 . Dashed line shows predicted power-law slope.

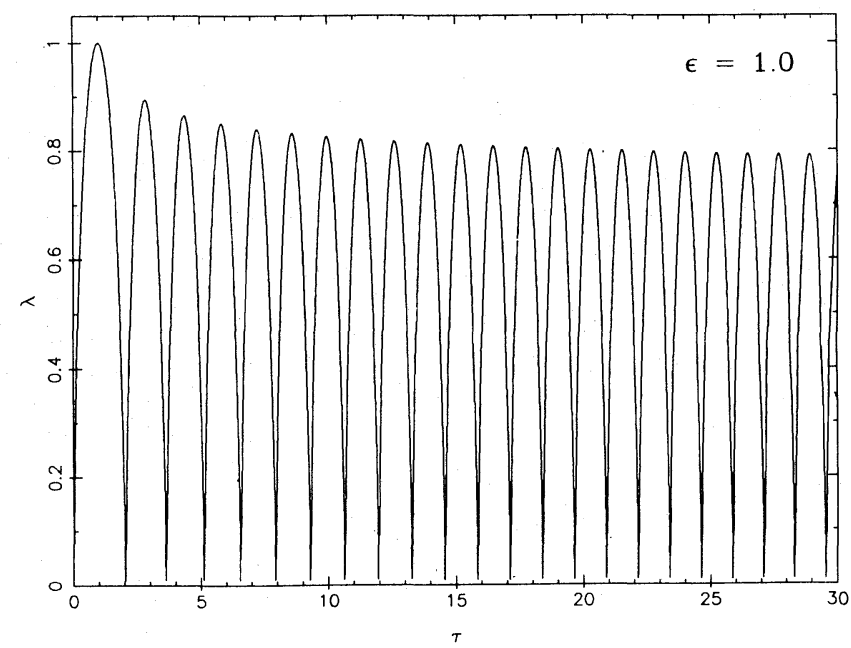

FIG. $9 b$ 


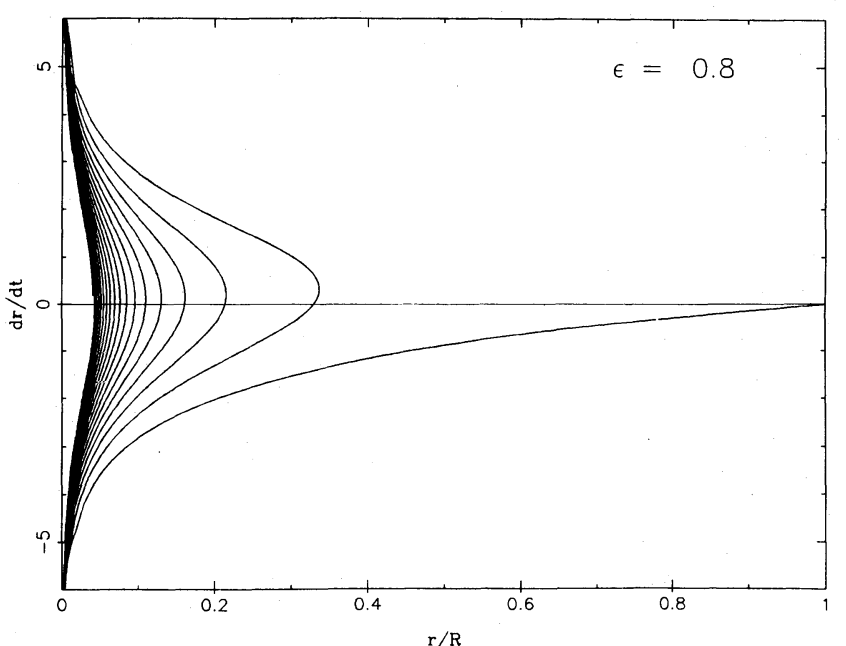

Fig. 10.-Spherical symmetry: instantaneous location of all particles in phase space for $\epsilon=0.8$.

each sequence shows the simultaneous location of all particles on the $d x / d t-x$ plane and demonstrates the existence of a denumerably infinite set of points along the phase plane curve at which $\partial x\left(t, M_{i}\right) / \partial M_{i}=0$. These points are associated with the density spikes seen in the third figure of each sequence. The spikes are contributed by particles which are approaching apapsis, with the exception that the first apapsis passage, turnaround, is not associated with a spike. The infinite-density spikes are truncated in the figures because the density is averaged over bins. The fourth and final figure in each sequence displays $\log (\mathscr{M})$ versus $\log (x / X)$ and illustrates the power-law nature of the mass profiles.

By and large, the detailed numerical solutions confirm the predictions of the asymptotic analytic theory which are summarized in equations (39). The largest discrepancy is that the value of $\gamma$ obtained from the analytic theory does not accurately estimate the rate at which $\mathscr{M}$ increases with $x$. This may be seen by comparing the slopes of the $\log (\mathscr{M})$ versus $\log$ $(x / X)$ plots with the theoretically predicted power laws given by the dashed lines.

\section{COMPARISON WITH PREVIOUS CALCULATIONS}

\section{a) Planar Simulations}

Other planar similarity solutions may be compared with numerical calculations done by Melott.(1983), who used a onedimensional cloud-in-cell method to simulate the large-scale clustering of 10,000 collisionless particles. Melott began his calculation at redshift $z=10,000$ and terminated it at $z=0$. His initial density perturbation was produced by a sinusoidal variation of amplitude $10^{-3}$ in the spacing of the particles. The entire system extended over one wavelength. Our similarity solutions demand different initial conditions. Nevertheless, they display the same qualitative features found in Melott's simulations. For example, compare the $d x / d t$ versus $x$ plot shown in our Figure 2 with Melott's Figure $8 a$.

\section{b) Spherical Simulations}

Interest in galaxy formation stimulated many investigations of spherically symmetric gravitational collapse. Early studies attempted to account for the approximate $\rho \propto r^{-3}$ distribution of the luminous material in elliptical galaxies. It was found that

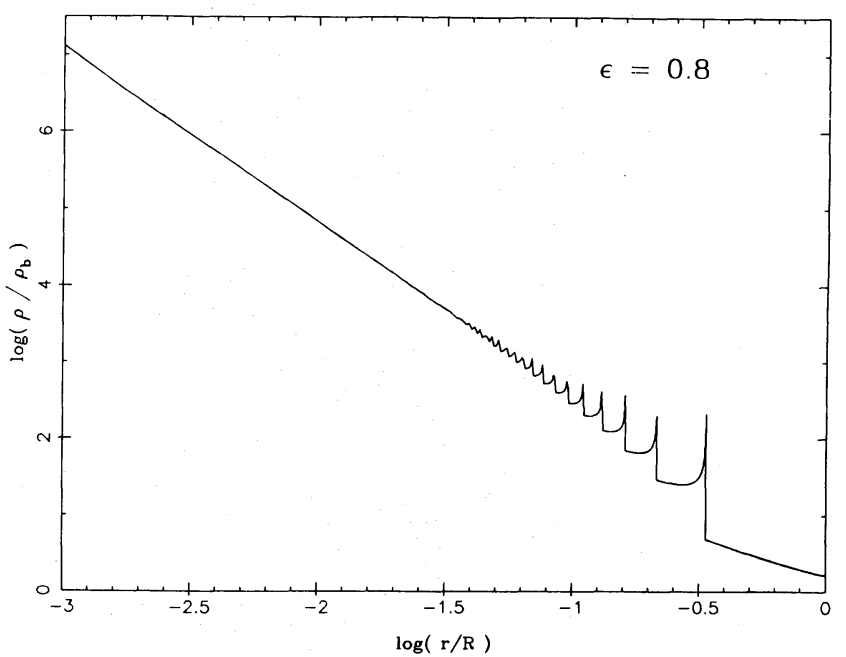

Fig. 11.-Spherical symmetry: ratio of actual to background density for $\epsilon=0.8$.

the collapse of an initially static, uniform-density sphere resulted in a final configuration with $\rho \propto r^{-3}$ (Henon 1964; Gott 1973). The secondary infall of bound but initially expanding material onto a collapsed core was discussed by Gunn and Gott (1972). Gott (1975) made the first attempt to determine a final density profile due to secondary infall. He considered a central overdense core embedded in an Einstein-de Sitter universe, essentially our $\epsilon=1$ case. Gott predicted that the asymptotic density profile would have $\rho \propto r^{-9 / 4}$. However, his numerical simulations produced $\rho \propto r^{-2.8}$. The extended flat rotation curves of spiral galaxies (Rubin, Ford, and Thonnard 1980; Krumm and Salpeter 1980) imply the presence of halos with $\rho \propto r^{-2}$. This led Gunn (1977) to extend Gott's study to more general initial-mass profiles in an attempt to discover conditions compatible with a final halo with $\rho \propto r^{-2}$. Gunn's analytic treatment is in some respects similar to the asymptotic theory presented in $\S$ III of our paper. However, he explicitly assumed that each particle's apapsis reaches a final value which is a fixed fraction of the turnaround radius. We find this

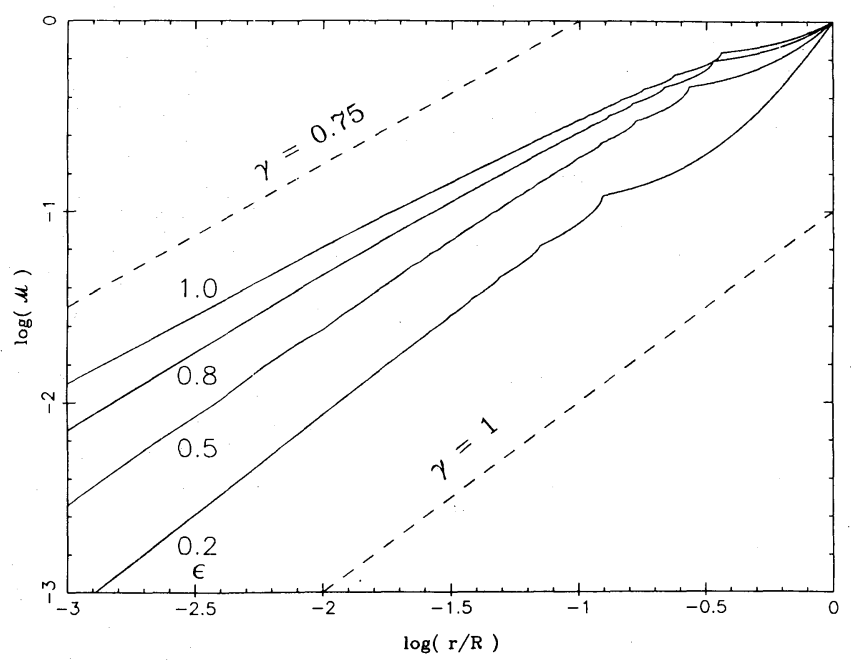

FIG. 12.- Spherical symmetry: profile of mass per unit solid angle for several $\epsilon$-values. Dashed lines show predicted power-law slopes for $\epsilon<\frac{2}{3}$ and for $\epsilon=1.0$ 
assumption to be invalid for $\epsilon<\frac{2}{3}$, precisely the range for $\epsilon$ for which the asymptotic theory predicts $\gamma=1$.

The evolution of an initially uniform-density region of finite radius surrounding a collapsed core was investigated by Dekel, Kowitt, and Shaham (1981) using a three-dimensional $N$-body code and by Pryor (1982) using a spherically symmetric code. Neither group produced models displaying extended flat rotation curves, although the Dekel et al. results provided some support for Gunn's (1977) predictions. However, these investigations were based on initial perturbations whose fractional excess masses $\delta$ decrease rather rapidly with increasing $M_{i}$; a relatively large fraction of the excess mass is contained in the collapsed cores as a consequence of the small radii at which the surrounding regions of uniform density are truncated. We find similarity solutions compatible with extended flat rotation curves for small $\epsilon$; these correspond to initial configurations for which $\delta$ decreases slowly as $M_{i}$ increases. Of course, our similarity solutions include continuous infall. It remains to be seen how extended the flat rotation curves are in truncated versions of low- $\epsilon$ models.

Support for this research was provided by the National Science Foundation through a graduate fellowship awarded to J. A. F. and through grant 80-20005.

\section{APPENDIX}

We determine the turnaround time $t_{*}$ and turnaround distance $x_{*}$ in terms of $t_{i}, x_{i}$, and $\delta$. The calculations are straightforward since a particle does not cross the orbits of other particles before it reaches turnaround.

\section{a) Planar Symmetry}

The equation of motion reads

$$
\frac{d^{2} z}{d t^{2}}=\frac{4}{9} \frac{z}{t^{2}}-4 \pi G M(z, t)
$$

Inserting equations (5) and (12) into equation (9) yields

$$
\frac{d^{2} z}{d t^{2}}=\frac{4}{9} \frac{z}{t^{2}}-\frac{2 z_{i}}{3(1-\delta) t_{i}^{2}}\left(\frac{t_{i}}{t}\right)^{4 / 3}
$$

for $t<t_{*}$. Solving the linear equation (A2) subject to the initial condition of unperturbed Hubble expansion, we obtain

$$
\frac{z}{z_{i}}=\frac{1}{(1-\delta)}\left\{\left(\frac{t}{t_{i}}\right)^{2 / 3}-\frac{\delta}{5}\left[3\left(\frac{t}{t_{i}}\right)^{4 / 3}+2\left(\frac{t}{t_{i}}\right)^{-1 / 3}\right]\right\} .
$$

From equation (A3), we find that to lowest order in $\delta \ll 1$,

$$
\frac{z_{*}}{z_{i}}=\frac{5}{12 \delta}, \quad \frac{t_{*}}{t_{i}}=\left(\frac{5}{6 \delta}\right)^{3 / 2} .
$$

The equation of motion is written

$$
\text { b) Cylindrical Symmetry }
$$

$$
\frac{d^{2} \varpi}{d t^{2}}=\frac{\varpi}{2 t^{2}}-\frac{2 G}{\varpi} M(\varpi, t)
$$

Inserting equations (6) and (12) into equation (9) yields

$$
\frac{d^{2} \varpi}{d t^{2}}=\frac{\varpi}{9 t^{2}}-\frac{1}{3(1-\delta)} \frac{\varpi_{i}^{2}}{t_{i}^{2} \varpi}\left(\frac{t_{i}}{t}\right)^{2 / 3},
$$

for $t<t_{*}$. Equation (A6) is nonlinear, and we have not been able to solve it analytically. However, numerical integrations imply that

$$
\frac{\varpi_{*}}{\varpi_{i}}=\frac{0.74}{\delta}, \quad \frac{t_{*}}{t_{i}}=\left(\frac{1.39}{\delta}\right)^{3 / 2} .
$$

The equation of motion simplifies to

c) Spherical Symmetry

$$
\frac{d^{2} r}{d t^{2}}=-\frac{4 \pi}{3} G \rho_{b}(t) r-\frac{G}{r^{2}} \delta M(r, t)=-\frac{G}{r^{2}} M(r, t)
$$

Since $M(r, t)$ is constant for $t<t_{*}$,

$$
\frac{d^{2} r}{d t^{2}}=-\frac{2 r_{i}^{3}}{9(1-\delta) t_{i}^{2}} \frac{1}{r^{2}}
$$


Although equation (A9) is nonlinear, it has an energy integral. We evaluate the energy constant by requiring that the velocity satisfy the unperturbed Hubble relation at $t=t_{i}$. This procedure yields

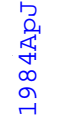

The turnaround parameters, to lowest order in $\delta \ll 1$, are

$$
\left(\frac{d r}{d t}\right)^{2}=\frac{2}{9(1-\delta)}\left(\frac{r_{i}}{t_{i}}\right)^{2}\left(\frac{r_{i}}{r}-\delta\right) .
$$

$$
\frac{r_{*}}{r_{i}}=\frac{1}{\delta}, \quad \frac{t_{*}}{t_{i}}=\frac{3 \pi}{4 \delta^{3 / 2}} .
$$

\section{REFERENCES}

Centrella, J., and Melott, A. L. 1983, Nature, 305, 196.

Dekel, A., Kowitt, M., and Shaham, J. 1981, Ap. J., 250, 561.

Frenk, C. S., White, S. D. M., and Davis, M. 1983, Ap. J., 271, 417.

Gott, J. R. 1973, Ap. J., 186, 481. 1975, Ap. J., 201, 296.

Gunn, J. E. 1977, Ap. J., 218, 592.

Gunn, J. E., and Gott, J. R. 1972, Ap. J., 176, 1.

Henon, M. 1964, Ann. d'Ap., 27, 83.
Klypin, A. A., and Shandarin, S. F. 1983, M.N.R.A.S., 204, 891

Krumm, N., and Salpeter, E. E. 1980, A.J., 84, 1138.

Melott, A. L. 1983, Ap. J., 264, 59.

Peebles, J. G. E. 1980, The Large-Scale Structure of the Universe (Princeton: Princeton University Press), $\$ \$ 6-8$.

Pryor, C. P. 1982, Ph.D. thesis, Harvard University.

Rubin, V. C., Ford, W. K., Jr., and Thonnard, N. 1980, Ap. J., 238, 471.

Zel'dovich, Ya. B. 1970, Astr. Ap., 5, 84

J. A. Fillmore: Robinson 105-24, Caltech, Pasadena, CA 91125

P. Goldreich: 170-25 So. Mudd, Caltech, Pasadena, CA 91125 